\title{
Guest Editorial: The Ethics of Reviewing
}

Papers submitted to the Journal of Dental Research are subjected to careful scientific review to the benefit of both readers and authors. Readers are thus assured that papers published in this journal have been scrutinized by scientists familiar with the subject material. Authors receive the reviewers' comments that in many instances improve final presentation and data analysis.

Reviewers, therefore, have particular responsibilities to the readers, to the authors, and to the field as a whole. Theirs is the responsibility to suggest acceptance or rejection, but they should not do so on trivial grounds or simply because they agree or disagree with the conclusions. Theirs is the responsibility to inspect the tables with care and, while suggesting improvements, to refrain from redirecting the investigation or demanding new and different approaches.

The papers that reviewers receive are in effect privileged communications, and they must be careful not to abuse their privilege. Before the actual publication of a paper, the author's rights must be respected, and no reviewer should delay review or return to give himself a publication advantage, or to protect one of his pending grants, or to give colleagues a competitive headstart.

If the reviewer knows of citations missed, he has the responsibility to cite them. If he finds an incorrect citation, he has the duty to point it out. If there are problems of investigative design or of sampling or of statistical analysis, the good reviewer provides enough detail so that the authors at least know where they went astray. And reviewers may indicate that particular graphs or charts or photomicrographs could be improved, extended or, in some instances, should be deleted.

Just as reviewers have responsibilities to authors, authors have responsibilities to reviewers. Authors must realize that the average review is based on a more detailed reading of their pa- pers than most readers will have time to make. If something is unclear to a reviewer, it is likely to be unclear to the average reader. Even if one reviewer is entirely favorable and another quite negative, it is worthwhile to consider the negative reviewer's comments. Adverse comments may be unpalatable, but they may also be the most useful comments of all.

Both reviewers and authors are responsible for some part of the present publication lag. Sitting on a review may deny an author or au. thors priority in publication. The practice is improper even if not deliberate. Failure of authors to respond to reviewers' comments may increase the backlog of papers in process. Editors may well set deadlines for manuscript revision so as to minimize the accumulation of manuscripts in effective limbo.

There are, of course, anomalies in the review. ing process. In some instances three tried and true scientific reviewers may reject a manuscript that three others might accept, or three may accept a paper that a second triad would reject. There are ideas that are scientifically premature and studies that are valid but postmature. Through writing and rewriting and rewriting again, an unacceptable manuscript may become an accepted one. By and large, however, the process of manuscript review has benefited the progress of science. Reviewers and writers have their responsibilities and their intellectual standards. When a manuscript is submitted to the Journal of Dental Research, all of these respon. sibilities come into play.

$$
\begin{aligned}
& \text {-Stanley M. Garn } \\
& \text { University of Michigan }
\end{aligned}
$$

The invitation to submit guest editorials brought about this contribution from Stanley Garn of the University of Michigan.

Our next issue may include one or two special interest articles. We will be interested in your reactions to them.

Barnet M. Levy, Editor

\section{Correction}

Dr. Robert M. Klein requests a correction in the abstract of his article in the July-August issue (55:611-616). The rats were 8 days old not 8 years. 